\title{
In vivo Morphometry of Menisci of the Knee in South Indians: A Preliminary Study
}

\author{
K.V.N. Dhananjaya ${ }^{1}$, B.V. Murlimanju ${ }^{2}$, Vinaya Poornima ${ }^{1}$, Sonali Ullal ${ }^{1}$, Prasanna Mitra ${ }^{3}$, \\ K.V.N. Dinesh ${ }^{4}$, Vasudha V. Saralaya ${ }^{2}$, Latha V. Prabhu ${ }^{2}$, K.U. Prashanth ${ }^{2}$
}

Background: Since the in vivo morphometric data on the menisci of the knee joint of Indian subjects are scarce, we hereby studied the width and thickness of the menisci using Magnetic Resonance Imaging (MRI) to establish standard dimensions of the normal medial and lateral meniscus as an aid to orthopaedic surgery.

Methods: Eighty menisci from 40 knee joints were analysed in each of three regions, the anterior horn, the mid body and the posterior horn. The thickness and width of the menisci were measured in sagittal and coronal T1-weighted and T2-weighted MRI images and analysed statistically.

Results: The mean thickness of medial meniscus at the anterior horn, mid body, and posterior horn were $6.3 \pm 1.1 \mathrm{~mm}$, $5.2 \pm 1.3 \mathrm{~mm}$, and $6.9 \pm 1.1 \mathrm{~mm}$, respectively. The respective values for the lateral meniscus were $4.8 \pm 0.7 \mathrm{~mm}$, $6.4 \pm 1.1 \mathrm{~mm}$, and $7.0 \pm 0.9 \mathrm{~mm}$. The mean width of medial meniscus at the anterior horn, mid body, and posterior horn were $10.5 \pm 1.2 \mathrm{~mm}, 7.8 \pm 1 \mathrm{~mm}$ and $13.9 \pm 0.9 \mathrm{~mm}$, respectively. The widths of lateral meniscus at the same regions were $11.8 \pm 1.4 \mathrm{~mm}, 8.6 \pm 1.2 \mathrm{~mm}$, and $12.0 \pm 0.9 \mathrm{~mm}$, respectively. The lateral meniscus was significantly wider than medial at the anterior horn and mid body $(p=0.00)$.

In contrast, the posterior horn of medial meniscus was significantly wider than lateral meniscus. Both menisci were significantly wider at their posterior horn, followed by the anterior horn and were significantly narrower at their mid body.

Conclusion: The present study provides new information on the meniscal thickness and width in South Indians that can be used in planning of orthopaedic and arthroscopic surgeries of the knee joint. However, the study needs to be analyzed with a large sample size for the better interpretation.

(Biomed J 2014;37:14-17)

Key words: menisci, meniscus, morphometry, thickness, width

$\mathrm{T}_{\mathrm{m}}^{\mathrm{s}}$ The knee menisci are a pair of wedge shaped semilunar fibrocartilage structures, which are attached to the periphery of the tibial plateau via the coronary ligaments, and to the femur via the meniscofemoral ligaments. ${ }^{[1]}$

\begin{abstract}
At a Glance Commentary
Scientific background of the subject

In allograft transplantation of the torn knee menisci, a mismatch would lead to the failure of the allograft or might end up in the degenerative changes. It is always good to have a set of anatomical morphometric parameters of the menisci like the width and thickness.
\end{abstract}

\section{What this study adds to the field}

The present study has added information on the meniscal thickness and width in South Indians which can be instrumental in the planning of orthopaedic and arthroscopic surgeries of the knee joint. The data of the present study are important to orthopaedic and arthroscopic surgeons, radiologists, anthropologists, and clinical anatomists.

\footnotetext{
${ }^{1}$ Department of Radiodiagnosis, Kasturba Medical College, Manipal University, Mangalore, India; ${ }^{2}$ Department of Anatomy, Centre for Basic Sciences, Kasturba Medical College, Manipal University, Mangalore, India; ${ }^{3}$ Department of Community Medicine, Kasturba Medical College, Manipal University, Mangalore, India; ${ }^{4}$ Department of Orthopedics, Kasturba Medical College, Manipal University, Mangalore, India.

Received: Sep. 11, 2012; Accepted: Jan. 17, 2013

Correspondence to: Dr. B.V. Murlimanju, Department of Anatomy, Kasturba Medical College, Manipal University, Bejai, Mangalore - 575 004, India. Tel: 91-824-2211746; Fax: 91-824-2421283; E-mail: flutemist@gmail.com
}

DOI: $10.4103 / 2319-4170.117889$

They deepen the articular surfaces of the tibia to improve the geometric fit with the femoral condyles ${ }^{[2]}$ and play an important role in knee stability, dynamic loading and for the maintenance of proper joint health ${ }^{[3]}$ Even though the 
success of meniscus transplantation depends critically on the precise sizing of the meniscal allograft, the accuracy of meniscal sizing can still be inadequate. ${ }^{[4]}$

It was reported that a set of parameters for describing the geometry of the medial and lateral menisci ${ }^{[5,6]}$ is essential as the mismatch might lead to failure of the allograft or subsequent development of degenerative changes. ${ }^{[7]}$ Also, some marked differences in the contour and insertion between the medial and lateral menisci are important determinants of meniscal injury. ${ }^{[8]}$ The normal meniscus demonstrates low signal on both T1 and T2 weighted images. Some studies report on the morphometric parameters of the cadaveric menisci, ${ }^{[9-11]}$ but the in vivo studies are scarce. Since the menisci perform important mechanical functions, they are exposed to injury. The meniscal tears might occur as part of a rotational trauma or bending, as the evolution of a joint degenerative process or an injury, or as a spontaneous injury resulting from a progressive structural failure without any correlation with trauma or a degenerative process. ${ }^{[1]]}$ It was reported that the variations of form and in particular, the thickness and width of the menisci can determine the possibility and kind of injury. ${ }^{[10]}$ In the literature, information or study on in vivo morphometry is not available from the South Indian population. Here, we report on the width and thickness of menisci of the knee joint in vivo using Magnetic Resonance Imaging (MRI). The purpose of present study was to establish the anatomic database on standard dimensions of the normal knee menisci in adults of South Indian population.

\section{METHODS}

Material is collected from a population of adult cohort of both genders. The MRI images of 80 menisci ( 40 medial and 40 lateral menisci from 40 knee joints) were analysed irrespective of their genders; and, MRIs of menisci with pathological changes like osteoarthritic changes were excluded. The study included only healthy adult subjects with no history of injury or abnormality. Informed consent was taken from each individual. The study has been approved by the Manipal University Ethics Committee. The age distribution of patients was between 20 to 40 years. The body height and weight of the individuals are not considered in the present study. MRI scans (1.5 Tesla MRI, Seimens Avento, Germany) were taken with the patients lying supine with the knees extended.

The images were viewed and the measurements performed using the SYNGOS software. Both the medial and lateral menisci were measured at three parts, the anterior horn, mid body, and posterior horn. The thickness and widths were measured at these three regions. The dimensions were studied in sagittal and coronal planes with T1-weighted acquisition and T2-weighted images [Figures 1-3]. The images which showed complete view of all parts of medial/lateral menisci are selected for this study. The measurements were performed at the middle of anterior horn, mid body and posterior horns. As the caliper used for measurement is inbuilt software, there was no difference in measured values. The measurements were performed by the same radiologist in all the scans. This has prevented the interobserver error. Statis-

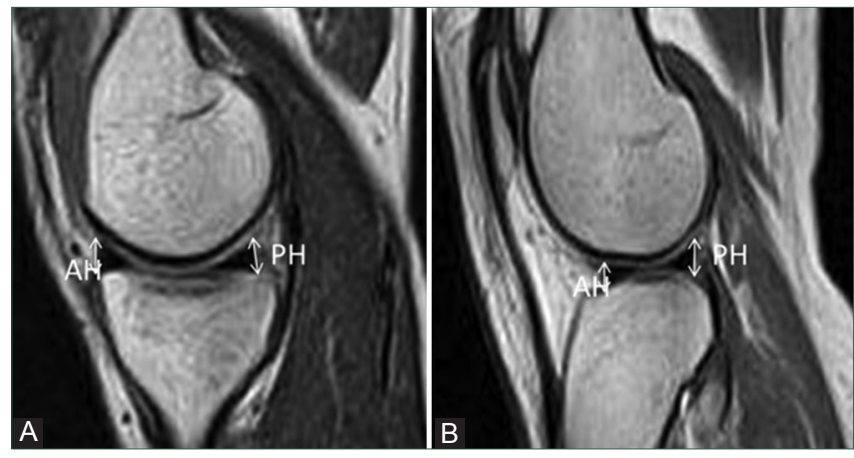

Figure 1: Sagittal Magnetic Resonance Imaging showing the thickness of the anterior horn $(\mathrm{AH})$ and posterior horn $(\mathrm{PH})$ of the medial meniscus (A) and lateral meniscus (B).

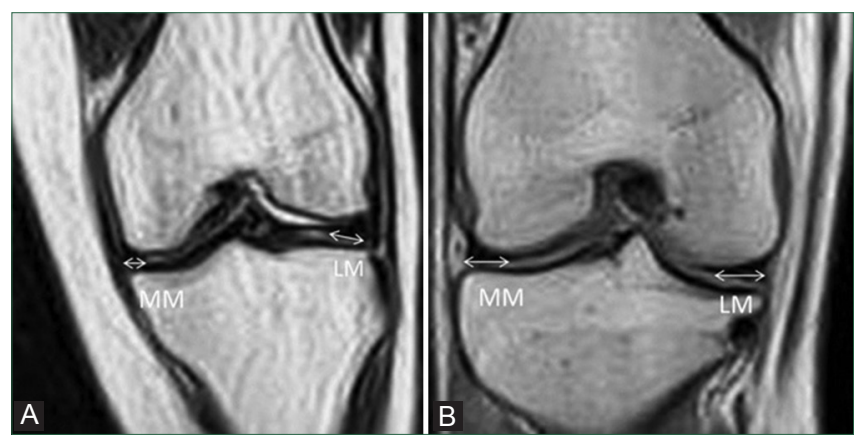

Figure 2: Coronal Magnetic Resonance Imaging showing the width of the anterior horn (A) and posterior horn (B) of medial (MM) and lateral menisci (LM).

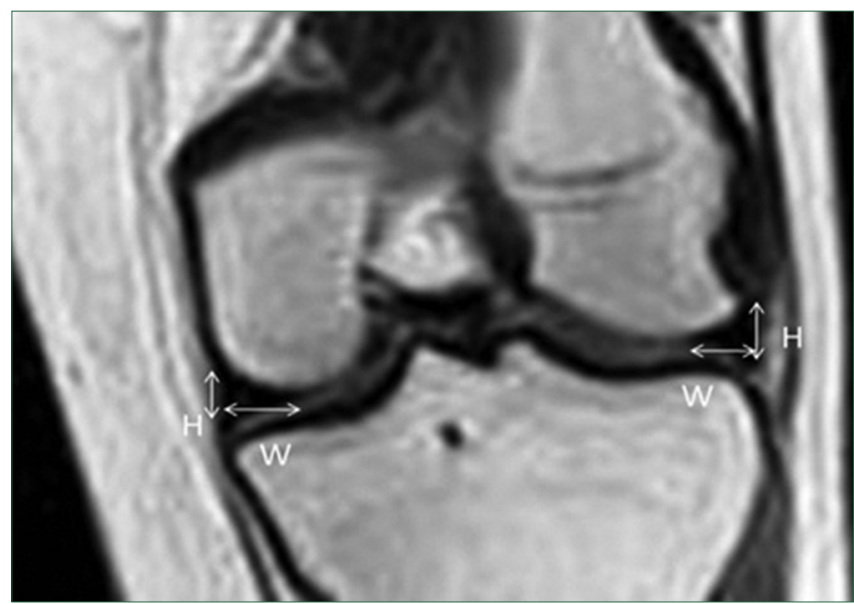

Figure 3: Coronal Magnetic Resonance Imaging showing the thickness $(\mathrm{H})$ and width $(\mathrm{W})$ of the medial and lateral meniscus at the mid body. 
tics were performed using Statistical Package for Social Sciences (SPSS) 15.0 software: Differences (mean \pm Standard Deviation, SD) between medial and lateral menisci were compared with the paired t-test; differences between the anterior horn, mid body, and posterior horn were analysed using Analysis of variance (ANOVA), and significance was set at $p \leq 0.05$.

\section{RESULTS}

The mean thicknesses of medial and lateral menisci at the anterior horn, mid body, and posterior horn are presented in Table 1 and the widths are presented in Table 2. In the sagittal plane, the thickness of the medial meniscus at the anterior horn was significantly more than that of the lateral meniscus, but the lateral meniscus was thicker than the medial meniscus at the mid body. The difference in thickness at the posterior horn was not significant $(p>0.05)$. Both the medial and lateral menisci were significantly thicker at their posterior horn than the anterior horn or mid body.

The lateral meniscus was significantly wider than medial at the anterior horn and mid body $(p=0.00)$. In contrast, the posterior horn of medial meniscus was significantly wider than lateral meniscus. Both menisci were significantly wider at their posterior horn, followed by the anterior horn, and were significantly narrower at their mid body.

\section{DISCUSSION}

In the medical research, intracapsular ligaments of the knee joint have been increasingly studied due to the invent of procedures like arthroscopy, computed tomography, and MRI, and because of their importance for clinical diagnosis and management. ${ }^{[12,13]}$ It was described that the size of meniscus is related to its function and any effort to replace the torn meniscus is assisted by the morphometric data. ${ }^{[14]}$ Prodromos et al.,$^{[15]}$ proposed the contralateral meniscal measurement as

Table 1: Thickness (mean \pm standard deviation, $\mathrm{mm}$ ) of menisci $(n=80)$ at different regions

\begin{tabular}{lcc}
\hline & Medial meniscus & Lateral meniscus \\
\hline Anterior horn* & $6.3 \pm 1.1^{*}$ & $4.8 \pm 0.7^{*}$ \\
Mid body* & $5.2 \pm 1.3^{*}$ & $6.4 \pm 1.1^{*}$ \\
Posterior horn & $6.9 \pm 1.1^{*}$ & $7.0 \pm 0.9^{*}$ \\
\hline$* p<0.05$, paired $t$ test and Analysis of variance (ANOVA)
\end{tabular}

Table 2: Width (mean \pm standard deviation, $\mathrm{mm}$ ) of menisci $(n=80)$ at different regions

\begin{tabular}{lcc}
\hline & Medial meniscus & Lateral meniscus \\
\hline Anterior horn* & $10.5 \pm 1.2^{*}$ & $11.8 \pm 1.4^{*}$ \\
Mid body* & $7.8 \pm 1^{*}$ & $8.6 \pm 1.2^{*}$ \\
Posterior horn* & $13.9 \pm 0.9^{*}$ & $12.0 \pm 0.9^{*}$ \\
\hline$* p<0.05$, paired $t$ test and Analysis of variance (ANOVA)
\end{tabular}

${ }^{*} p<0.05$, paired $t$ test and Analysis of variance (ANOVA) the gold standard for sizing the menisci before transplantation, which has started a trend to use plain radiographs and MRI of meniscus of the contralateral knee. ${ }^{[13]}$

Variations of form, and in particular, the width and thickness of the menisci can determine the likelihood and severity of injury. ${ }^{[10]}$ According to Smillie, ${ }^{[8]}$ the lateral meniscus occupies a larger area of the tibial plateau, due to its semicircular form and lesser interhorn distance. According to a cadaveric study by Braz and Silva, ${ }^{[11]}$ there were no significant differences in width between the anterior horn, posterior horn, and the mid body of the lateral meniscus; however, the medial meniscus proved to be the widest in the posterior horn, followed by the mid body and anterior horn. This was confirmed by Almeida et al.,${ }^{[10]}$ who observed that the posterior horn of the medial meniscus was wider than the lateral meniscus in cadavers, but did not find any statistically significant difference between the medial and lateral menisci with respect to the mid body. However, the lateral meniscus was wider than the medial at the anterior horn. In the present study, both menisci were wider at their posterior horn, followed by the anterior horn and narrower at their mid body. We also observed that the lateral meniscus was wider than medial meniscus at the anterior horn and mid body, whereas the medial meniscus was wider than lateral meniscus at the posterior horn.

Braz and Silva ${ }^{[11]}$ reported that the posterior horn of medial meniscus was the thinnest followed by the anterior horn and the mid body. In the lateral meniscus, anterior horn was the thinnest followed by the posterior horn and mid body. In contrast, Almeida et al. ${ }^{[10]}$ reported no significant difference among the thickness at the anterior horn, mid body and posterior horn in the medial meniscus, and that the lateral meniscus was the thickest at the mid body and while the medial meniscus was thicker than the lateral at the anterior and posterior horns, the lateral meniscus thicker than the medial meniscus at the mid body. In the present study, the medial meniscus was thicker than the lateral meniscus at the anterior horn, whereas the lateral meniscus was thicker than the medial meniscus at the mid body and the thickness of the two menisci was not significantly different at the posterior horn. Both the medial and lateral menisci were thicker at their posterior horn than the anterior horn or mid body.

Our findings are similar to those of Erbagci et al., ${ }^{[2]}$ which is also an in vivo MRI study. In a study on the location of meniscal ruptures, Rico and Ayala ${ }^{[16]}$ observed that the medial meniscus is more commonly affected, and the lesions were more frequent at the mid body followed by the posterior and anterior horns. On the other hand, Braz and Silva ${ }^{[11]}$ pointed out that the wider the meniscus, the more susceptible it is to injuries, presumably because of the higher exposure to the actions of the femoral condyles. They reported that the anterior horn is the narrowest part of 
the medial meniscus and the mid body was the thickest part of both menisci, yet the mid body of both menisci presented the highest meniscal tears. We interpret the discrepancies in our observations with these authors as due to differences in measurements in cadaveric menisci vs. in vivo MRI measurements; and some racial differences may also account for some of the differences.

Since availability of accurate morphometric data is crucial for restoring the normal joint conditions through meniscal tissue engineering and transplantation, ${ }^{[17]}$ the new information on the meniscal thickness and width in South Indians provided by the present study can be instrumental in the planning of orthopaedic and arthroscopic surgeries of the knee joint.

We believe that, a definite conclusion cannot be drawn from the present study. The limitations of the study include small sample size of the data studied. The present study should be compared with a large sample size from the South Indian population. Future implications of the study include relating the meniscal size with the height and weight of the individual.

\section{ACKNOWLEDGEMENTS}

The corresponding author is grateful to Juli Valtschanoff, MD, the Professor and Chair of Anatomy, Human Structure and Function Department, American University of Antigua College of Medicine, University Park, Saint John's, Antigua, West Indies for the constant advice and support.

\section{REFERENCES}

1. Kurosaka M, Yagi M, Yoshiya S, Muratsu H, Mizuno K. Efficacy of the axially loaded pivot shift test for the diagnosis of a meniscal tear. Int Orthop 1999;23:271-4.

2. Erbagci H, Gumusburun E, Bayram M, Karakurum G, Sirikci A. The normal menisci: In vivo MRI measurements. Surg Radiol Anat 2004;26:28-32.

3. Chivers MD, Howitt SD. Anatomy and physical examination of the knee menisci: A narrative review of the orthopedic literature. J Can Chiropr Assoc 2009;53:319-33.

4. Yoon JR, Kim TS, Wang JH, Yun HH, Lim H, Yang JH. Importance of independent measurement of width and length of lateral meniscus during preoperative sizing for meniscal allograft transplantation. Am J Sports Med 2011;39:1541-7.

5. Pollard ME, Kang Q, Berg EE. Radiographic sizing for meniscal transplantation. Arthroscopy 1995;11:684-7.

6. Haut TL, Hull ML, Howell SM. Use of roentgenography and magnetic resonance imaging to predict meniscal geometry determined with a three-dimensional coordinate digitizing system. J Orthop Res 2000;18:228-37.

7. Dienst M, Greis PE, Ellis BJ, Bachus KN, Burks RT. Effect of lateral meniscal allograft sizing on contact mechanics of the lateral tibial plateau: An experimental study in human cadaveric knee joints. Am J Sports Med 2007;35:34-42.

8. Smillie IS. Injuries of the knee Joint, $4^{\text {th }}$ ed. London: Living Stone; 1975.

9. Murlimanju BV, Nair N, Pai SR, Pai MM, Gupta C, Kumar V, et al. Morphometric analysis of the menisci of the knee joint in South Indian human foetuses. Int J Morphol 2010;28:1167-71.

10. Almeida SK, De Moraes AS, Tashiro T, Neves SE, Toscano AE, De Abreu RR. Morphometric study of menisci of the knee joint. Int J Morphol 2004;22:181-4.

11. Braz PR, Silva WG. Meniscus morphometric study in humans. J Morphol Sci 2010;27:62-6.

12. Kale A, Kopuz C, Edyzer M, Aydin ME, Demyr M, Ynce Y. Anatomic variations of the shape of the menisci: A neonatal cadaver study. Knee Surg Sports Traumatol Arthrosc 2006;14:975-81.

13. Murlimanju BV, Nair N, Kumar B, Krishnamurthy A, Gupta C, Chethan P. Clinically oriented morphometric study of medial and lateral menisci of the knee joint in adult cadavers. Clin Ter 2012;163:105-8.

14. Stone KR, Stoller DW, Irving SG, Elmquist C, Gildengorin G. 3D MRI volume sizing of knee meniscus cartilage. Arthroscopy 1994;10:641-4.

15. Prodromos CC, Joyce BT, Keller BL, Murphy BJ, Shi K. Magnetic resonance imaging measurement of the contralateral normal meniscus is a more accurate method of determining meniscal allograft size than radiographic measurement of the recipient tibial plateau. Arthroscopy 2007;23:1174-9.e1.

16. Rico EG, Ayala CE. Localización de lasrupturas meniscales en nuestro médio. Rev Mex Ortop Traumato 1997;11:10-3.

17. Bloecker K, Wirth W, Hudelmaier M, Burgkart R, Frobell R, Eckstein F. Morphometric differences between the medial and lateral meniscus in healthy men: A three dimensional analysis using magnetic resonance imaging. Cells Tissues Organs 2012;195:353-64. 